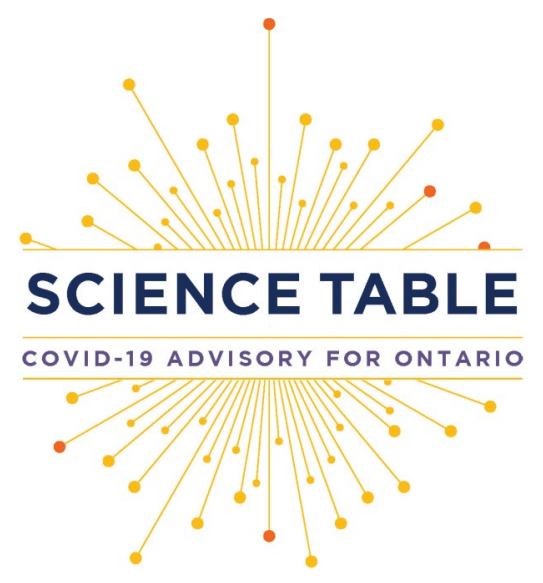

Version 1.1

Published: March 5, 2021

Updated on March 7, 2021. Version 1.0 is available under Additional Resources at https://doi.org/10.47326/ocsat.2021.02.12.1.0.

Citation: Presseau J, Desveaux L, Allen U, et al. Behavioural science principles for supporting COVID-19 vaccine confidence and uptake among Ontario health care workers. Science Briefs of the Ontario COVID-19 Science Advisory Table. 2021;2(12). https:// doi.org/10.47326/ocsat.2021.02.12.1.0

Author Affiliations: The affiliations of the members of the Ontario COVID-19 Science Advisory Table can be found at https:// covid19-sciencetable.ca/.

Declarations of Interest: The declarations of interest of the members of the Ontario COVID-19 Science Advisory Table can be found at https://covid19-sciencetable.ca/.

About Us: The Ontario COVID-19 Science Advisory Table is a group of scientific experts and health system leaders who evaluate and report on emerging evidence relevant to the COVID-19 pandemic, to inform Ontario's response. Our mandate is to provide weekly summaries of relevant scientific evidence for the COVID-19 Health Coordination Table of the Province of Ontario, integrating information from existing scientific tables, Ontario's universities and agencies, and the best global evidence. The Science Table summarizes its findings for the Health Coordination Table and the public in Science Briefs.

The Behavioural Science Working Group is a group of scientific experts and public health leaders with specific expertise in behaviour change. Their expertise spans behavioural medicine, health, clinical and social psychology, behavioural economics, and implementation science. The Working Group evaluates emerging scientific evidence related to vaccination in healthcare workers and the general population, alongside other recommended protective behaviours such as physical distancing, mask-wearing, testing, and supported quarantine. The Working Group reports its findings to the public and the Science Table. Its findings are also
SCIENCE BRIEFS

\section{Behavioural Science Principles for Supporting COVID-19 Vaccine Confidence and Uptake Among Ontario Health Care Workers}

Justin Presseau, Laura Desveaux, Upton Allen, Trevor Arnason, Judy L. Buchan, Kimberly M. Corace, Vinita Dubey, Gerald A. Evans, Leandre R. Fabrigar, Jeremy M. Grimshaw, Anne Hayes, Julian House, Douglas G. Manuel, Robert J. Reid, Robert Steiner, Ashini Weerasinghe, Brian Schwartz, on behalf of the Ontario Behavioural Science Working Group and the Ontario COVID-19 Science Advisory Table

\section{Key Message}

Health Care Workers (HCWs) are the backbone of Ontario's COVID-19 pandemic response and are a key vaccination priority group. About $80 \%$ of Ontario HCWs intend to receive COVID-19 vaccine. ${ }^{1}$

Challenges include the logistics of delivering the vaccine to this mobile and diverse group and improving vaccine confidence in the remaining $20 \%$. These challenges can be overcome by allaying safety concerns and highlighting personal benefits; tailoring messages to factors associated with lower intention (e.g. age, gender, ethnicity and work setting); employing trusted leaders to set the tone and peers to build social norms; and leveraging public health organizations and health institutions as existing channels of influence.

\section{Background}

HCWs are the backbone of Ontario's COVID-19 pandemic response and are a key vaccination priority group. Ontario's healthcare organizations and public health units are developing and launching campaigns to support HCWs to get vaccinated. Vaccination is a health behaviour and the behavioural sciences have contributed much to understanding vaccination decisions and uptake in the general public and HCWs. $^{2-8}$

\section{Question}

Which behavioural science principles can be used to support vaccine confidence and uptake in health care workers?

\section{Findings}

Figure 1 summarizes evidence-based approaches to support Ontario organizations, including employers, professional associations, unions, and others in maximizing voluntary vaccination among Ontario's HCWs. These approaches draw from wellestablished behavioural science principles. 
Correspondence to: Secretariat of the Ontario COVID-19 Science Advisory Table (info@covid19-sciencetable.ca)

Copyright: 2021 Ontario COVID-19 Science Advisory Table. This is an open access document distributed under the terms of the Creative Commons Attribution License, which permits unrestricted use, distribution, and reproduction in any medium, provided that the original work is properly cited.

The views and findings expressed in this Science Brief are those of the authors and do not necessarily reflect the views of all of the members of the Ontario COVID-19 Science Advisory Table, its Working Groups, and its partners.

Communication
$\begin{gathered}\text { Principles } \\ \text { Conal }\end{gathered}$

Figure 1. Nine Principles for Supporting Vaccination Confidence and Uptake in Health Care Workers

\section{General Communication Principles}

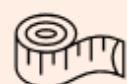

One size does not fit all
Tailoring: Authentically work with $\mathrm{HCW}$ communities to address barriers and enablers to vaccination in HCWs, which vary by gender, age, ethnicity, rural/urban work location, and profession ${ }^{8}$. Trust is key for vaccine uptake.

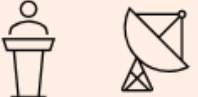
$\cdots$

Distinguish messenger (who), channel (how) and message (what)
Messenger: Involve people/organizations that Ontario HCWs trust including hospitals, unions, and health professionals. Find messengers who are similar (occupation, background) to the HCWs being supported to get vaccinated.

Channel: Use consistent messaging across multiple channels \& formats to maximize reach.

Message: Use the principles below to optimise messaging to address vaccination barriers.

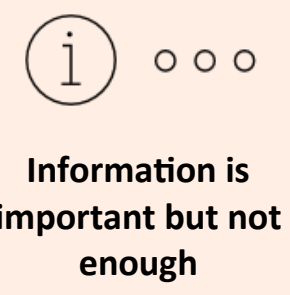

Beyond information/education: Vaccination uptake is based on many factors. Information and education are unlikely to maximize vaccine uptake in the absence of other supports.

Beyond how vaccines work: Combine with why rapid and safe development was possible.

Reconsider Terminology: Consider reframing discussions on 'vaccine hesitancy' to promoting vaccine 'confidence' or 'acceptance' while addressing uncertainties and concerns. 

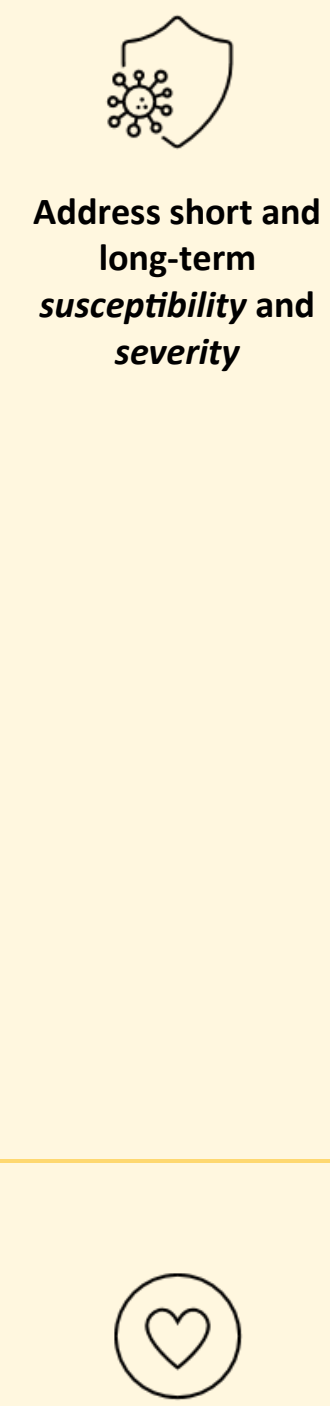

Understand and validate emotions
Address vaccine side effect severity and susceptibility: Identify main vaccine side effects, their severity (e.g., mild, moderate, or severe) and their prevalence. Clarify which side effects indicate that an immune response is happening (a good thing). Frame data around the number of people who don't experience these effects. Clarify that allergic reactions are rare, and highlight supports in place to manage them if they occur.

Underscore that COVID-19 is a much greater risk than COVID-19 vaccines: Use a direct comparison of the risk of vaccine side effects with the risk of contracting COVID-19 and developing serious issues.

Address short and long-term COVID-19 vaccine side effects: Clarify that side effects are almost always shortterm by showing how many days/weeks after vaccine people report side effects. Create a system to enable $\mathrm{HCWs}$ to report and receive real-time support on vaccine side effect questions to demonstrate responsibility and reassure that support is available if side effects are experienced.

Explain speed of vaccine development: Explain that common vaccine development bottlenecks include lack of funding and difficulty recruiting participants to clinical trials. COVID-19 vaccines build on 15+ years of vaccine research (i.e., not from scratch), had lots of funding and the world's best scientists, governments, universities and pharmaceutical companies who immediately mobilized their resources in pursuit of protecting the world from COVID-19. Clarify that because so many volunteered, large trials ran more quickly than usual because they could rapidly recruit large samples worldwide.

Explain speed of vaccine approval: Health Canada is independent, and its mandate includes ensuring the safety of vaccines for Canadians. COVID-19 vaccines received priority in the review process, whereas vaccines for less transmissible diseases are required to wait in a queue. Health Canada shortened the administrative and organizational processes, but the safety standards were just as strict as for any other vaccine.

Pair empathy with action: Normalize feelings of anxiousness and uncertainty but make sure to pair empathy with actions that can help to address emotions.

Promote hope: Instill optimism by connecting vaccination to promoting safety for friends and family and supporting the return to normal. 


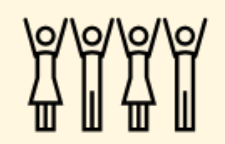

Build positive social norms through social comparison
Visibility of similar people: Identify the peers/colleagues of HCWs who have similar lived and work experience, and who have decided to receive the COVID-19 vaccine. Promote and amplify those individuals willing to champion their decisions to receive the COVID-19 vaccine (e.g., tally boards with photos; online).

Provide data: Provide regular data to HCWs on how many HCWs have been vaccinated and highlight increases over time if/when uptake accelerates. Use local data whenever possible.

Framing: Build positive social norms by emphasizing the \% of HCW who have vaccine-positive views; reporting on \% who are hesitant risks a 'backfire effect' of creating a negative social norm. Acknowledge hesitancy but frame positively by highlighting available supports to address concerns.

\section{Principles for Supporting HCWs Once They Have Decided to Get Vaccinated}

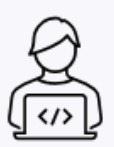

\section{Support motivated \\ HCWs while they wait}

Setup an online registry: Establish a central registry to enable pro-active registration, which could also enable regular updates to stay informed of vaccine eligibility and timing and remind HCWs of the need for two doses (if relevant). Ensure that registration and associated communication efforts promote equitable access (cf. communication principles).

Make it easy: Communicate where (e.g., provide map), eligibility (who and why); defaults (e.g., consider establishing an opt-out vs opt-in system is acceptable); when (e.g., how appointment fits with shifts); how often (when to return for 2 nd dose, if applicable); reminders (e.g., text message); clarify if financial supports are available to support vaccination including paid time and transportation to get vaccinated and additional paid sick leave in case of vaccine side effects resulting in missed time from work.

Make it as easy as possible to get vaccinated

Streamline consenting: Optimize the consent process (e.g., complete forms ahead of time, consider whether written vs. verbal consent is needed) and remove barriers within consent forms that risk deferring or delaying vaccination.

Clarify all steps: Clarify all steps needed to get vaccinated and who is eligible and when. 


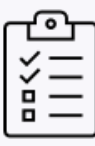

Address the gap between intention and action by creating clear plans
Support use of implementation intentions: Supplement the scheduling process for both doses (where relevant) by providing HCWs with the capacity to select where and when to get vaccinated and encourage them to record the date, time and location of vaccination (implementation intention), to be reinforced by a formal reminder system (e.g., email, text).

Encourage public commitment: Encourage communicating vaccine intention to foster social accountability and build norms.

Anticipate and plan for obstacles: Work with HCWs to anticipate and address barriers that could impede them personally getting vaccinated and collective vaccination progress.

An Evidence Synthesis Briefing Note ${ }^{8}$ on vaccine uptake among HCWs is also available for additional information.

\section{Interpretation}

Organisations across Ontario are developing and delivering strategies to build vaccine confidence and support voluntary COVID-19 vaccination among Ontario healthcare workers. The behavioural science principles outlined in this brief represent effective behavioural strategies based on a synthesis of available evidence. Organisations and groups responsible for communications and vaccination logistics can leverage behavioural science through the application of these principles to inform new campaigns and enhance existing ones with approaches that leverage what is known about vaccination decision making, behaviour, and underlying beliefs.

\section{References}

1. Desveaux L, Savage R, Tadrous M, et al. Beliefs Associated with Intentions of Non -Physician Healthcare Workers to Receive the COVID-19 Vaccine in Ontario, Canada. Infectious Diseases (except HIV/AIDS); 2021. https:// doi.org/10.1101/2021.02.19.21251936

2. Brewer NT, Chapman GB, Rothman AJ, Leask J, Kempe A. Increasing Vaccination: Putting Psychological Science Into Action. Psychol Sci Public Interest. 2017;18 (3):149-207. https://doi.org/10.1177/1529100618760521

3. World Health Organization. Behavioural Considerations for Acceptancy and Uptake of COVID-19 Vaccines: WHO Technical Advisory Group on Behavioural Insights and Science for Health. World Health Organization; 2020. Accessed February 1, 2021. https://www.who.int/publications/i/item/9789240016927

4. Corace KM, Srigley JA, Hargadon DP, et al. Using behavior change frameworks to improve healthcare worker influenza vaccination rates: A systematic review. Vaccine. 2016;34(28):3235-3242. https://doi.org/10.1016/j.vaccine.2016.04.071

5. Corace K, Garber G. When knowledge is not enough: Changing behavior to change vaccination results. Hum Vaccines Immunother. 2014;10(9):2623-2624. https://doi.org/10.4161/21645515.2014.970076

6. Jacobson Vann JC, Jacobson RM, Coyne-Beasley T, Asafu-Adjei JK, Szilagyi PG. 
Patient reminder and recall interventions to improve immunization rates. Cochrane Effective Practice and Organisation of Care Group, ed. Cochrane Database Syst Rev. Published online January 18, 2018. https:// doi.org/10.1002/14651858.CD003941.pub3

7. Konnyu K, Benitez G. What Are the Barriers and Facilitators to Individuals' Willingness to Be Vaccinated for COVID-19? Brown University; 2020. https:// www.brown.edu/public-health/cesh/news/2021/01/what-are-barriers-andfacilitators-individuals'-willingness-be-vaccinated-covid-19

8. Evidence Synthesis Network. COVID-19 Vaccine Uptake among Health Care Workers. COVID-19 Evidence Synthesis Network; 2021. https://esnetwork.ca/ briefings/covid-19-vaccine-uptake-among-health-care-workers/ 